\title{
Mammography in Management of Breast Lesions
}

W. B. JAMES,* M.B., CH.B., F.F.R. ; R. W. IRVINE, $†$ M.B., CH.B., F.R.C.S.ED.

\begin{abstract}
Cummary : Comparison of the clinical and mammography findings in 101 histologically proved breast lesions showed that the accuracy of each method in diagnosing carcinoma was about $90 \%$, but when taken together an accuracy of $98 \%$ was reached. Though mammography was less accurate in the diagnosis of benign lesions, it was found to be a valuable complement in the management of a lesion in the large atrophic breast and for showing an impalpable carcinoma. In mammography of the young dense breast a negative result must be interpreted with caution.
\end{abstract}

\section{Introduction}

Soft-tissue radiography of the breast (mammography) is a technique which uses the inherent radiographic contrast of the breast tissues to show pathological signs. Salomon (1913) seems to have been the first to use radiological methods in studying breast tumours when he made radiographs of mastectomy specimens. The method was used preoperatively by a few enthusiasts, but did not gain any general acceptance until the publication of work by Leborgne (1953), Ingleby and Gershon-Cohen (1960), and Egan (1960). In this country Samuel and Young (1964) have been largely responsible for the resurgence of interest in the method. The most important reasons for this renewal of interest are the lack of improvement in prognosis in breast cancer and improved radiographic techniques leading to the possibility of diagnosis at a stage when the disease is not clinically apparent (Young, 1968). Opinion has not been unanimous, however, about the value of mammography, and recently Thomas and McDonald (1967) have cast doubts on the accuracy of the method.

The present report concerns two years' experience with mammography in the investigation of patients with breast complaints. Simultaneously with this clinical investigation a survey of well women was conducted, the details of which are reported elsewhere (Irvine and James, 1969).

\section{Patients}

In 251 patients aged 19 to 83 mammography was carried out. The presenting feature was in most patients a lump in the breast, in some pain, and in a few discharge from the nipple. A small number were examined because of anxiety arising from a bad family history of breast cancer. Nearly all were referred from the surgical outpatient clinics, a few from general practitioners.

\section{Description of Radiographic Technique}

The technique used is that described by Egan (1964) and Samuel and Young (1964). The kilovoltage used is in the range 25 to 40 derived from a Machlett HD $40 x$-ray tube without added filtration. Two exposures at right angles are made in the cranio-caudad and medio-lateral projections on fine-grain industrial film using a double film technique (Bohrer, 1964). A long copper cone is used to cut down scattered radiation. It is $\frac{\rho}{\vec{\phi}}$ essential to observe these details of technique in order to demon- $\varrho$ strate differences in density within the breast tissue and the is minute calcifications observed in many cases of carcinoma. All $\vec{O}$ films were processed manually, reported on by the radiologist, and subsequently reviewed with the surgeon.

\section{Radiographic Signs}

Mammographic appearances in the normal breast at different $ळ$ ages, in mammary dysplasia, and in benign and malignant ${ }_{-}^{+}$ tumours of the breast have been extensively documented, of particularly by Egan (1964). The breast in a young nulliparouso woman commonly shows a homogeneous density, and it is difficult or impossible to distinguish the shadow of a mass $\vec{\omega}$ within this density. Consequently, mammography is of ten less valuable, and a negative result may be misleading in this group ${ }_{\mathbb{\Phi}}$ of patients. As the woman becomes older more adipose tissue 3 is deposited, and in the post-menopausal atrophic breast the $\mathbb{\Phi}^{-}$ soft-tissue opacity of a lesion becomes more easily discernible ${ }_{-}$ on the mammogram. The radiographic signs which may be seen include a soft-tissue opacity which will vary in density. and outline according to its nature, the presence and appearance of fine calcifications, and alteration in breast structure such as nipple retraction, skin thickening, and vessel asymmetry.

\section{Results}

Mammography was carried out on 251 women with breast complaints. In 101 patients a histological diagnosis was obtained and the clinical and mammography findings in these patients are reviewed. In the remaining 150 women either no lesion was found clinically or radiographically or a presumptive benign lesion is being followed up.

The clinical and radiological diagnoses in 55 cases of proved carcinoma are compared in Table I. It was not always clearo from the case-notes or the request card what the surgeon's clinical diagnosis was. Where the surgeon had clearly stated? that he was doubtful or where a frozen section had been carried N out before mastectomy the clinical diagnosis was considered to $>$ be equivocal. It can be seen from Table I that a correct pre-O operative diagnosis was made by the surgeon in 37 patients, ann equivocal diagnosis in 12, and that in 6 out of the 55 then surgeon was wrong. This gives a clinical true positive rate ${ }^{1 \omega}$ of $67 \%$, or if equivocal diagnoses are considered correct of nearly $90 \%$. Similarly, the radiological true positive rate is almost $82 \%$, or if equivocal diagnoses are considered correctos just over $90 \%$. The mammographic diagnosis was wrong on five occasions. Further study of Table I indicates clearly that clinical and mammographic diagnoses are complementary. Thus in two patients in whom no lesion could be demonstrated on the mammogram the carcinoma was clinically obvious. In two patients with no palpable lesion a carcinoma was demonstrated on the mammogram. One of these patients had veryo large breasts and presented with pain (Fig. 1). In the other? patient a symptomless carcinoma was found in the contralateralo breast. In a third patient with breast pain in whom no lesion?

1 True positive rate is the percentage of proved cancers correctly diagnosed preoperatively. 
was palpable the mammogram was reported as normal, but in retrospect a small area of skin-thickening and a very small carcinoma were seen (Fig. 2).

TABLE I.-Comparison of Clinical and Radiological Diagnoses in 55 Carcinomas

\begin{tabular}{c|c|c|c|c|c|c}
\hline & \multicolumn{5}{|c}{ Radiological Diagnosis } \\
\hline \multirow{4}{*}{$\begin{array}{c}\text { Clinical } \\
\text { diagnosis }\end{array}$} & Carcinoma & Equivocal & Benign & Normal & Total \\
\cline { 2 - 6 } & Equivocal & 33 & 2 & - & 2 & 37 \\
\cline { 2 - 6 } & Benign & 2 & 3 & 1 & - & 12 \\
\cline { 2 - 6 } & Normal & 2 & - & - & 1 & 3 \\
\cline { 2 - 6 } & Total & 45 & 5 & 2 & 3 & 55 \\
\hline
\end{tabular}

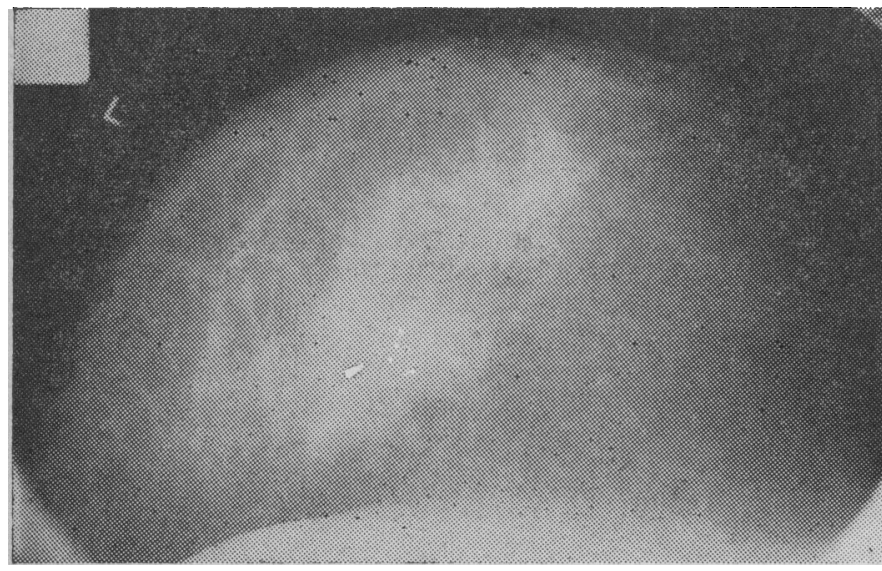

Fig. 1.- Woman aged 68. Breast pain. No mass palpable. Mammogram - deep carcinoma with typical calcifications (retouched) in large breast.

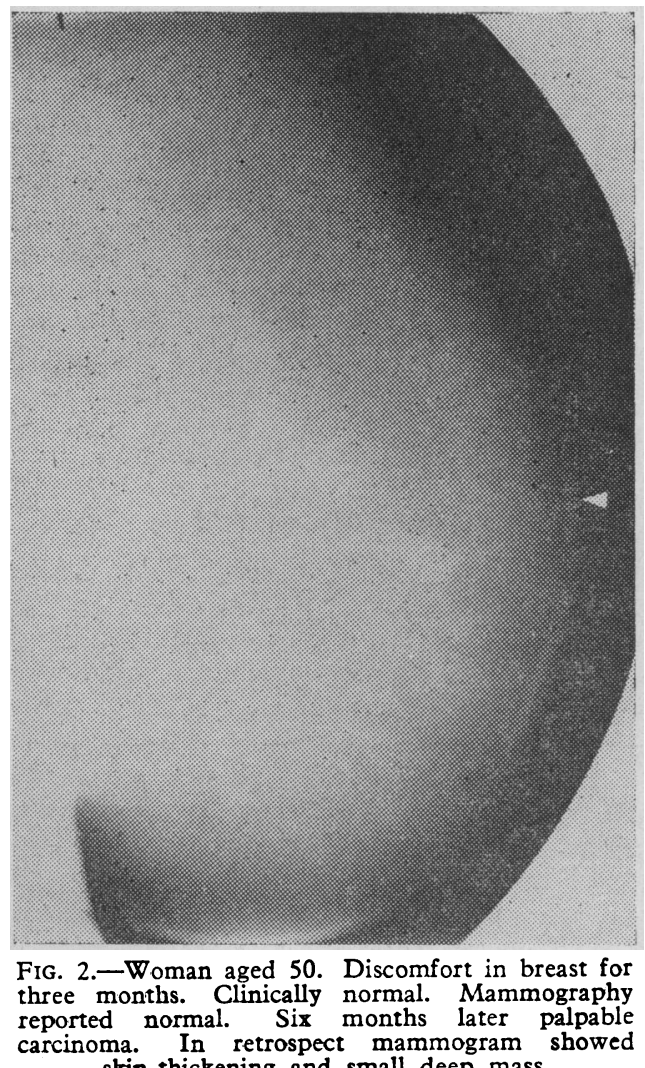

skin-thickening and small deep mass.

The clinical and radiological diagnoses in 46 cases with histologically benign lesions are compared in Table II. The surgical

- True negative rate is the percentage of proved benign lesions correctly diagnosed preoperatively. opinion was correct in 32 cases for a true negative rate $^{2}$ of nearly $70 \%$ while the radiological true negative rate was only $54 \%$. In fact, no lesion could be demonstrated on the mammogram in 10 patients. This was due mainly to the difficulty of localizing lesions in young patients with dense breasts. On the other hand an incorrect diagnosis of carcinoma was made in only two patients radiologically and in four patients clinically.

TABLE II.-Comparison of Clinical and Radiological Diagnoses in 46 Benign Lesions

\begin{tabular}{c|c|c|c|c|c|c}
\hline & \multicolumn{5}{|c}{ Radiological Diagnosis } \\
\hline \multirow{3}{*}{$\begin{array}{c}\text { Carcinoma } \\
\text { Clinical }\end{array}$} & Equivocal & Benign & Normal & Total \\
\cline { 2 - 6 } & Equinosis & 1 & 1 & 2 & - & 4 \\
\cline { 2 - 6 } & Benign & - & 2 & 6 & 2 & 10 \\
\cline { 2 - 7 } & Normal & - & - & - & - & - \\
\hline & Total & 2 & 9 & 25 & 10 & 46 \\
\hline
\end{tabular}

The incidence of the commoner radiological findings in these 101 lesions is shown in Table III. As might be expected, an irregular mass was defined on the mammogram in the majority of carcinomas, but in about 1 in 5 the mass was well defined. Fortunately in most of the well-defined carcinomas the presence of other signs such as calcification or skin thickening allowed a diagnosis of carcinoma to be made. In two patients with carcinoma no lesion could be demonstrated on the mammogram even in retrospect. When an opacity was seen in the mammogram of a patient with a benign lesion this was usually well defined, but nearly a quarter showed an irregular contour and in two patients there was associated skin thickening. About one-third of the carcinomas showed multiple tiny calcifications of the type described as typical of carcinoma (Fig. 3). Calcifications seen in association with benign lesions were usually fewer in number and coarser. Vascular asymmetry was found to be an unreliable sign in this series.

TABLE III.-Comparison of Radiological Findings in Benign and Malignant Lesions

\begin{tabular}{|c|c|c|c|}
\hline \multirow{2}{*}{\multicolumn{2}{|c|}{ Mammographic Sign }} & \multicolumn{2}{|c|}{ Lesion } \\
\hline & & Carcinoma & Benign \\
\hline $\begin{array}{l}\text { Irregular or ill-defined mass } \ldots \\
\text { Skin-thickening } \\
\text { Calcification associated with mass } \quad \ldots \\
\text { Well-defined mass } \\
\text { Vascular asymmetry associated with } \\
\text { Vass }\end{array}$ & $\begin{array}{l}\cdots \\
\cdots \\
\cdots \\
\cdots\end{array}$ & $\begin{array}{r}42 \\
31 \\
18 \\
10 \\
6\end{array}$ & $\begin{array}{r}8 \\
2 \\
5 \\
28 \\
7\end{array}$ \\
\hline
\end{tabular}

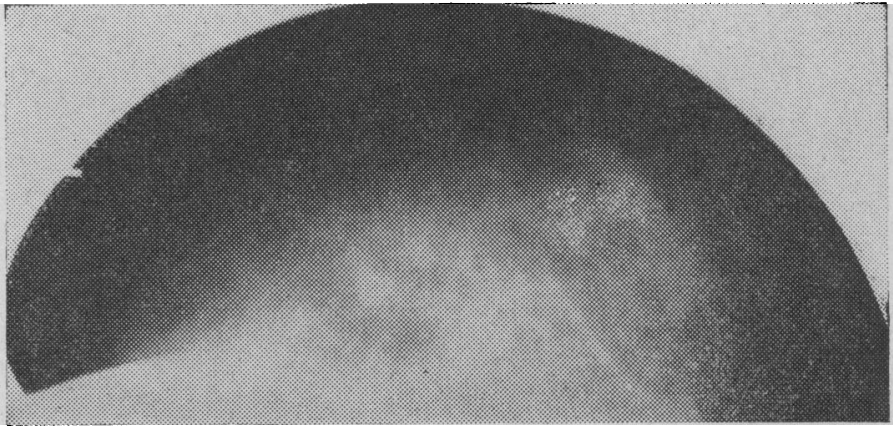

FIG. 3.-Clinically-irregular mobile lump in breast. Mammogramtypical multiple tiny calcifications of carcinoma.

\section{Discussion}

Our experience with mammography tends to confirm the encouraging reports of other workers. In the series of 55 carcinomas reported in this paper only one lesion was actually missed by both the surgeon and the radiologist, and even this case, which occurred early in the series, should with more experience have been diagnosed radiologically. There is no 
doubt that an increase in diagnostic accuracy is possible by using both methods together. Mammography is capable of demonstrating an impalpable lesion. Even with the most careful technique, however, mammography will still miss a significant and irreducible number of lesions and must not be used as a substitute for clinical examination. In our experience mammography is likely to miss carcinoma in the young dense breast or in the bulky invasive type of lesion, poorly differentiated from the surrounding tissues and showing no secondary signs such as calcification or skin-thickening. Such a lesion is usually clinically obvious.

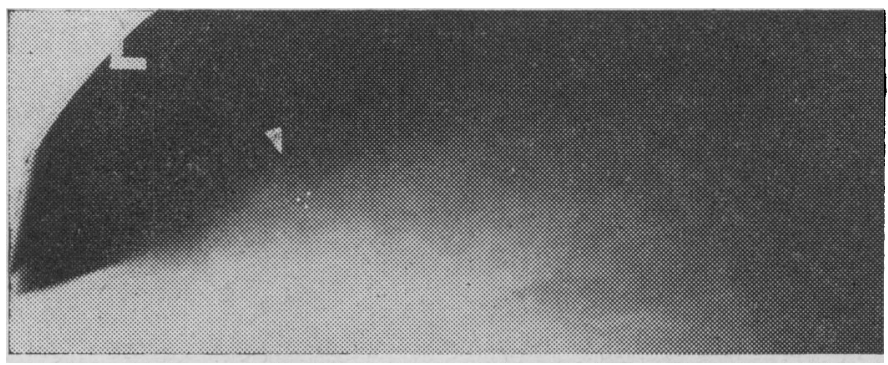

FIG. 4.-Clinically-oval lump in breast, probably fibroadenoma. Mammogram-well-defined mass 6 by $3 \mathrm{~cm}$. with $1 \mathrm{~cm}$. irregular density showing tiny calcifications at lateral aspect-benign lesion + carcinoma. Histology-large area fibroadenosis with $1 \cdot 5-\mathrm{cm}$. scirrhous carcinoma.

In the palpable lesion mammography may allow a positive diagnosis of carcinoma to be made preoperatively or, conversely, may point to a benign lesion. Thus in some of the carcinomas considered to be equivocal by the surgeon the evidence of the mammogram permitted mastectomy to be carried out without resort to preliminary biopsy. Less frequently, in a benign lesion considered to be carcinoma or equivocal by the surgeon the mammogram may indicate the need for biopsy rather than mastectomy. It cannot be stressed too strongly that mammography does not as yet replace the need for biopsy, and this is particularly true in the younger age groups where mammography is less accurate (Copland and Scott, 1968). Even now a confident radiographic diagnosis of carcinoma may support the clinical diagnosis sufficiently to obviate biopsy in elderly or infirm patients in whom the primary treatment is to be by palliative radiotherapy, hormones, or cytotoxic drugs. Mammography may also be helpful in a patient with known or suspected benign disease where a carcinoma may be demonstrated $\stackrel{0}{-}$ within an area of fibrocystic disease (see Fig. 4). The mammogram can also direct the pathologist's attention to the relevanto area of the surgical specimen in such cases.

Thomas and McDonald (1967) sounded a cautionary note $\overrightarrow{\bar{F}}$ on the accuracy of mammography. They reported only ao $61 \%$ true positive rate in the detection of proved carcinoma, and they also found that Egan has not maintained his initialo high accuracy. The majority of their patients, however, seem to have been in the younger age groups, and there is no doubt that accuracy in mammography increases with the age of the $e^{\infty}$ patient. It is possible that discrepancies in accuracy between $\overrightarrow{0}$ reports reflect the varying incidence of patients in younger age groups.

Further improvements in technique will increase the value of mammography, and research on such improvements must be stimulated and supported (Fitts, 1966). Mammographyir must be used only as a complement to clinical examination ando not as a substitute, otherwise the method will be brought into+ disrepute.

\section{REFERENCES}

Bohrer, S. P. (1964). British fournal of Radiology, 37, 237. Copland, M. M., and Scott, W. G. (1968). American fournal of Surgery 116, 57

Egan, R. L. (1960). Radiology, 75, 894.

Egan, R. L. (1964). Mammography. Springfield, Illinois, Thomas.

Fitts, W. T. (1966). Surgery, Gynecology and Obstetrics, 122, 1077. 通

Ingleby, H., and Gershon-Cohen, J. (1960). Comparative Anatomy, Pathology and Roentgenology of the Breast. Philadelphia, University of Pennsylvania Press.

Irvine, R. W., and James, W. B. (1969). In press.

Leborgne, R. A. (1953). The Breast in Roentgen Diagnosis. Montevideo, Impresora Uruguya.

Salomon, A. (1913). Archiv für klinische Chirurgie, 101, 573.

Samuel, E., and Young, B. (1964). Clinical Surgery, edited by C. Robญ and R. Smith, p. 431. London, Butterworths.

Thomas, M. A., and McDonald, E. J. (1967). Medical Annals of the District of Columbia, 36, 468 .

Young, G. B. (1968). Fournal of the Royal College of Surgeons of $\frac{0}{3}$ Edinburgh, 13, 12 .

\section{Percutaneous Needle Nephrostomy}

C. S. OGG,* M.D., B.SC., M.R.C.P.; H. M. SAXTON, $\dagger$ M.R.C.P., F.F.R.; J. S. CAMERON, ${ }^{*}$ M.D., B.SC., M.R.C.P.

Cummary : Percutaneous nephrostomy is a simple tech$\checkmark$ nique for temporary drainage of an obstructed kidney. Under local anaesthesia a ureteric catheter is passed through a Vim-Silverman needle into the renal pelvis and is connected to a drainage bag. Seven cases (six successful) in which this method was used are described and the indications are discussed. It has been free from complications, is acceptable to patients, and is felt to represent a useful addition to the methods available for the treatment of obstruction of the upper urinary tract.

* Renal Physician, Guy's Hospital, London S.E.1.

† Radiologist, Guy's Hospital, London S.E.1.

\section{Introduction}

In the management of obstruction of the upper urinary tract it is sometimes desirable to provide temporary relief of the obstruction, usually until definitive treatment can be under- $\frac{\mathrm{D}}{\mathbb{D}}$ taken. Retrograde catheterization and operative nephrostomy $\stackrel{\rho}{\%}$

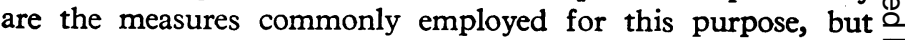
each has disadvantages. It may be impossible to pass a ureteric catheter above the obstructing lesion; even when passed it is 8 uncomfortable and often becomes dislodged downward into the bladder. On the other hand, the surgeon may hesitate to undertake nephrostomy because it involves a relatively major procedure requiring a general anaesthetic for what may be a transitory obstruction. Percutaneous nephrostomy is a simple 